\section{P497 A META-SYNTHESIS OF MEN WHO HAVE SEX WITH MEN (MSM)'S EXPERIENCES AND PERCEPTIONS OF HUMAN PAPILLOMAVIRUS (HPV) VACCINATION}

${ }^{1}$ Lewis Clarke*, 'Janette Pow, ${ }^{1}$ Sheona Mchale, ${ }^{2}$ Brian Williams. ${ }^{1}$ Edinburgh Napier University, School of Health and Social Care, Edinburgh, UK; ${ }^{2}$ Edinburgh Napier University, School of Health and Social Care, Sighthill Court, UK

\subsection{6/sextrans-2019-sti.579}

Background Men who have Sex with Men (MSM) are disproportionately burdened by diseases attributed to the Human Papillomavirus (HPV) when compared to other male populations. High uptake of this vaccine is crucial for anal cancer and genital wart pevention. Although female-oriented vaccination programmes indirectly extend HPV vaccination to heterosexual males through herd immunity, these confer little to no benefit for MSM. Previous (predominantly quantitative) studies exploring HPV-MSM perceptions have reported MSM's a lack of awareness/understanding of HPV and its relation their health, not being offered the vaccine by a healthcare professional, and varied perceptions of vaccination may affect uptake. The complex nature of HPV vaccination decision-making is not reflected in these studies. We aimed to conduct a qualitative systematic review of studies exploring MSM experience, perceptions, and attitudes toward HPV infection and vaccination.

Methods Last updated in January 2019, 6 databases were searched for studies using qualitative methods and reporting experiences/perceptions of MSM regarding HPV infection and vaccination. MSM quotes and author's interpretations of data were extracted from primary studies. Thematic synthesis was used to develop analytical themes.

Results 9 papers (reporting 8 studies) were included. The thematic synthesis identified two analytical themes: (1) Vaccination content and delivery and (2) Addressing patient-level stigma. Theme 1 explores the varied experiences of how (and to what extent) healthcare provider's recommendation of the vaccine plays a role in the decision-making process. Theme 2 explores the influence and subsequent implications of MSM's perceptions and experiences of stigma and how these relate to vaccine initation in broader health systems.

Conclusion MSM vaccination across many developed countries continues to be a new phenomenon. By understanding more about the unique mechanisms which underpin MSM's vaccination behaviour, in collaboration with an understanding of the particular health systems which vaccination is implemented in, we can better design and co-produce intervientions to inform and enhance uptake.

Disclosure No significant relationships.

\section{P499 HUMAN PAPILLOMAVIRUS DETECTION IN RESIDUAL SAMPLES FROM STI TESTS IN MEN WHO HAVE SEX WITH MEN}

${ }^{1}$ Diarmuid Nugent, ${ }^{1}$ Oliver Stirrup, ${ }^{1}$ Sarah Pett, ${ }^{2}$ Simon Beddows, ${ }^{2}$ Marta Checchi, ${ }^{2}$ David Mesher, ${ }^{2}$ Kavita Panwar, ${ }^{3}$ Kate Soldan, ${ }^{1}$ Richard Gilson*. 'University College London, Institute for Global Health, London, UK; ${ }^{2}$ Public Health England, London, UK; ${ }^{3}$ Public Health England, HIVISTI, London, UK

\subsection{6/sextrans-2019-sti.580}

Background Anorectal swab specimens, either alone, or pooled with first catch urine (FCU) and pharyngeal swab specimens, are used to test for STIs in MSM. The residual sample, after routine testing, may be used to monitor human papillomavirus (HPV) prevalence in this population, but the sensitivity of HPV detection in such specimens is unknown.

Methods MSM attending a UK sexual health clinic were consented to collect additional specimens to compare the detection of HPV in a dedicated swab, with detection in a residual anorectal and/or pooled specimen. All subjects provided 3 specimens: (i) anorectal swab (for chlamydia(CT)/gonorrhoea (GC) testing); (ii) pooled anorectal/pharyngeal/FCU specimen (for GC/CT); (iii) dedicated anorectal swab for HPV. Specimen (iii) and residual material from specimens (i) and (ii) were tested for type-specific HPV DNA (19 confirmed/possible high-risk (HR) genotypes and genotypes 6/11). HPV detection was by in-house multiplex PCR and Luminex-based genotyping assay.

Results 129 MSM were recruited; mean age 38.1 years; 24\% were HIV-positive. 92/129 (71\%) had type-specific HPV DNA detected in $\geq 1$ specimen; 80/129 (62\%) had HR-HPV. 70/123 participants $(56.9 \%)$ with sufficient residual pooled specimen, and a dedicated HPV specimen had detectable HPV on both and $40(32.5 \%)$ were HPV-negative on both; overall concordance $89 \%$ (95\%CI 83,94). Prevalence in pooled samples was $4.1 \%(-1.9,10.0)$ higher than dedicated samples. $74 / 125$ participants $(59.2 \%)$ with sufficient residual anorectal specimen, and dedicated anorectal HPV specimen had detectable HPV on both and 36 (28.8\%) were HPV-negative on both; overall concordance $88 \%(81,93)$. Prevalence in residual samples was $5.6 \%(-0.6,11.8)$ higher than dedicated samples.

Conclusion Residual anorectal and pooled STI test specimens offer comparable sensitivity to anal HPV swab samples, which are typically used in prevalence studies. This supports use of residual samples to monitor HPV prevalence, as currently proposed in the UK to evaluate the impact of targeted MSM HPV vaccination.

Disclosure No significant relationships.

\section{P500 BARRIERS AND OPPORTUNITIES FOR PARTNER SERVICES RELATED TO SYPHILIS AMONG MEN WHO HAVE SEX WITH MEN IN ANCHORAGE, ALASKA}

${ }^{1}$ Laura Quilter*, ${ }^{2}$ Melissa Boyette, ${ }^{3}$ Julia Brennan, ${ }^{2}$ Jessica Harvill, ${ }^{2}$ Susan Jones, ${ }^{1}$ Eloisa Llata, ${ }^{2}$ Joe Mclaughlin, ${ }^{3}$ Bozena Morawski, ${ }^{1}$ Alison Ridpath, ${ }^{2}$ Tracy Smith, ${ }^{3}$ Amanda Tiffany, ${ }^{1}$ Elizabeth Torrone, ${ }^{4}$ Kyle Bernstein. ${ }^{1}$ Centers for Disease Control and Prevention, Division of STD Prevention, Atlanta, USA; ${ }^{2}$ Alaska Department of Health and Social Services, Division of Public Health, Anchorage, USA; ${ }^{3}$ Centers for Disease Control and Prevention, Division of Scientific Education and Professional Development, Epidemiology Workforce Branch, Atlanta, USA: ${ }^{4}$ Centers for Disease Control and Prevention, Atlanta, USA

\subsection{6/sextrans-2019-sti.581}

Background Early syphilis is highly infectious and can lead to life-threatening complications if left untreated. During 2017 2018, early syphilis cases in Alaska tripled from 24 to 76, with most 2018 cases occurring among Anchorage men who have sex with men (MSM). Partner services can mitigate transmission. We conducted an investigation to identify syphilis partner services barriers and opportunities among MSM in Anchorage.

Methods During October 19-November 13, 2018, we administered an anonymous survey to men and transgender women aged $\geq 18$ years residing in Alaska who reported sex with a man during the previous 6 months. The self-administered survey, available online and as paper, asked about knowledge, risk behaviors, medical care-seeking practices, and attitudes 
regarding syphilis. A convenience sample was recruited from digital platforms (e.g., Facebook ${ }^{\circledast)}$ and bars, clinics, and community-based organization events.

Results Of 119 survey respondents, 90 (76\%) lived in the Anchorage/Mat-Su region. Of these, $10.0 \%$ (95\% confidence interval [CI]: 5.3-17.9) reported a syphilis diagnosis during the previous 12 months and having a median of 3 (interquartile range: 1-6) sex partners during the previous 6 months. High-risk behaviors commonly associated with syphilis were reported, including condomless anal sex $(36.7 \%$; 95\% CI: 27.4-47.0) during the previous month, $\geq 1$ episode of group sex $(26.7 \%$; 95\% CI: $18.6-36.6)$ during the previous 6 months, and meeting sex partners online or on a geospatial mobile app (66.7\%; 95\% CI: 56.4-75.5) during the previous 6 months. Additionally, 44.4\% (95\% CI: 34.6-54.7) reported a sex partner living outside Anchorage and 31.1\% (95\% CI: 22.5-41.3) outside Alaska.

Conclusion Large numbers of sex partners outside Alaska presents barriers to partner services among Anchorage MSM with syphilis. Opportunities include using innovative strategies (e.g., technology-based partner services and out-of-jurisdiction partnerships) to reach populations at risk for syphilis.

Disclosure No significant relationships.

\section{P501 LOW PREVALENCE OF HIGH-RISK ANAL HPV IN YOUNG GAY AND BISEXUAL MALES AFTER THE UNIVERSAL HPV VACCINATION PROGRAM IN AUSTRALIA}

${ }^{1}$ Eric Chow*, ${ }^{2}$ Sepehr Tabrizi, ${ }^{1}$ Christopher Fairley, ${ }^{1}$ Rebecca Wigan, ${ }^{2}$ Alyssa Cornall, ${ }^{2}$ Steph Atchison, ${ }^{2}$ Dorothy Machalek, ${ }^{3}$ Jane Hocking, 'Catriona Bradshaw, ${ }^{2}$ Suzanne Garland, ${ }^{1}$ Marcus Chen. ${ }^{1}$ Alfred Health, Melbourne Sexual Health Centre, Carlton, Australia; ${ }^{2} T$ The Royal Women's Hospital, Centre for Women's Infectious Disease Research, Parkville, Australia; ${ }^{3}$ University of Melbourne, Melbourne School of Population and Global Health, Parkville, Australia

\subsection{6/sextrans-2019-sti.582}

Background Australia introduced a school-based quadrivalent human papillomavirus (HPV) vaccination program for females in 2007. This was extended to include boys aged 12-13 from 2013 , with a two-year catch-up for boys aged $\leq 15$. This study examined HPV prevalence among young gay and bisexual males (GBM) who were age-eligible for vaccination in the school-based program.

Methods Males aged 16-20 years were recruited from sexual health clinics and the community in Melbourne in 20172018 , if they reported any form of male sexual contact, and were residents of Australia from 2013. A clinician-collected anal swab, self-collected penile swab and oral rinse were collected and analysed for detection and 37 HPV genotypes (Roche Linear Array). Preliminary results from 114 GBM were analysed and full results will be available for presentation.

Results The mean age of GBM was 18.6 years (SD 1.0). The majority $(80 \%)$ were recruited from clinics and 20\% from the community. The median number of lifetime male partners was 10 [IQR 5-25] for receptive oral sex, four [IQR 1-11] for receptive anal sex and one for insertive anal sex [IQR 0-6]. Overall, 64\% received at least one dose of vaccine documented via the National HPV Vaccination Program Register. Prevalence of quadrivalent vaccine-preventable HPV genotypes was 4.9\% (95\% CI: $1.6-11 \%$ ) for anal, 3.4\% (95\% CI: $0.7-$ $9.5 \%)$ for penile and $0 \%$ (95\% CI: $0-3.2 \%$ ) for oral sites. Only two men, both unvaccinated, had high-risk vaccine- preventable HPV genotypes: one with anal HPV16 (1\%); the other penile HPV16 (1\%).

Conclusion Statistical analysis comparing before and after the male vaccination program will be performed until recruitment is completed. The preliminary analysis shows the prevalence of anal HPV 16/18 among young GBM following the schoolbased male HPV vaccination was low. The addition of male HPV vaccination to female programs may reduce the incidence of anal cancer among GBM.

Disclosure No significant relationships.

\section{P503 TRENDS IN AWARENESS AND USE OF PREP AMONG HIV-NEGATIVE MEN WHO HAVE SEX WITH MEN IN VANCOUVER, TORONTO, AND MONTREAL}

${ }^{1}$ Heather Armstrong*, ${ }^{2}$ Shenyi Pan, ${ }^{1}$ Justin Barath, ${ }^{3}$ Gbolahan Olarewaju, ${ }^{4}$ Ricky Rodrigues, ${ }^{5}$ Marc Messier-Peet, ${ }^{5}$ Herak Apelian, ${ }^{2}$ Mark Hull, ${ }^{6}$ Darrell Tan, ${ }^{7}$ Nathan Lachowsky, ${ }^{8}$ Jody Jollimore, ${ }^{4}$ Trevor Hart, ${ }^{9}$ Daniel Grace, ${ }^{5}$ Gilles Lambert, ${ }^{10}$ Joseph Cox, ${ }^{3}$ David Moore. ${ }^{1} B C$ Centre for Excellence in HIVIAIDS, Epidemiology and Population Health, Vancouver, Canada; ${ }^{2}$ British Columbia Centre for Excellence in HIVIAIDS, Vancouver, Canada; ${ }^{3} B C$ Centre for Excellence in HIVIAIDS, Vancouver, Canada; ${ }^{4}$ Ryerson University, Toronto, Canada; ${ }^{5}$ Direction Régionale de Santé Publique Montréal, Montreal, Canada; ${ }^{6}$ St. Michael's Hospital, Centre for Urban Health Solutions, Li Ka Shing Knowledge Institute, Toronto, Canada; ' University of Victoria, School of Public Health and Social Policy, Victoria, Canada; ${ }^{8}$ Community Based Research Centre, Vancouver, Canada; ${ }^{9}$ University of Toronto, Toronto, Canada; ${ }^{10}$ McGill University, Montreal, Canada

\subsection{6/sextrans-2019-sti.583}

Background Awareness, availability, and public funding of preexposure prophylaxis (PrEP) has increased substantially since it was approved by Health Canada in 2016 but policies and guidelines vary by province. Given the dynamic nature of PrEP policy and promotion, we sought to determine awareness and use of PrEP among gay, bisexual, and other men who have sex with men (gbMSM) in Vancouver, Toronto, and Montreal.

Methods Beginning in $02 / 2017$, sexually-active gbMSM $\geq 16$ years and living in Vancouver, Toronto, or Montreal were recruited into a cohort study using respondent-driven sampling (RDS). Data are included up to $08 / 2018$. At study visits every 6 months (Vancouver) or 12 months (Toronto, Montreal), participants completed a computer-assisted self-interview which included questions on PrEP awareness and use in the past 6 months (P6M). We used RDS-adjusted, general estimating equations accounting for two levels of clustering (RDS recruitment chain; participant) to evaluate temporal trends (monthly prevalence) of awareness and P6M usage of PrEP among HIVnegative participants.

Results 1619 HIV-negative gbMSM completed 2074 study visits (1205 Montreal, 285 Toronto, 584 Vancouver). Over the course of the study, PrEP awareness significantly increased among gbMSM in Montreal from 62.6\% during the first 6month period to $84.8 \%$ during the last 6-month period (OR:1.07, 95\%CI:1.02-1.12, per month); awareness remained consistent in Toronto (89.8\%-96.0\%, ns) and Vancouver (84.5\%-95.0\%, ns). Use of PrEP increased significantly in all three sites: Montreal, $10.5 \%$ during the first 6-month period to $30.9 \%$ during the last 6-month period (OR:1.06, 95\% CI:1.02-1.11); Toronto, $12.5 \%$ to $23.3 \%$ (OR:1.15, 95\% CI:1.02-1.30); Vancouver, $11.4 \%$ to $35.2 \%$ (OR:1.16, $95 \%$ CI:1.06-1.27).

Conclusion Awareness of PrEP appears to be $\geq 85 \%$ among HIV-negative gbMSM across all three cities. Even though 